\title{
Collaborative Mind Mapping Test Case Trasability Matrix Techniques
}

\author{
K. Rajasekaran, ${ }^{1}$, T.K.Nithyarao, ${ }^{2}$ \\ *(Department, of computer science Dhanraj Baid Jain College Chennai India) \\ Email: krs.salem@gmail.com \\ ** (Department of computer science, Mphil scholar Dhanraj Baid Jain College Chennai India) \\ Email nithyakrishnarao89@gmail.com
}

ABSTRACT : In the business world communitarian mind outline an extremely utilize full graph used to outwardly sort out data Visualizing. The Mind maps is an online electronic condition is an all the more effective approach to work together and upgrade cooperation, and the execution specifically when working from various areas and diverse circumstance. It is likewise an extremely extraordinary approach to introduce thoughts and ideas to potential clients. Online electronic personality mapping programming or desktop mind mapping programming with shared workspace and work bunches reconciliation furnishes clients with a cloud-based stage and devices which take into consideration multiuser altering continuously, making multi-provincial groups more compelling over the association. Mind maps or realistic coordinators can be priceless apparatuses for visual masterminds and visual students. The way toward making a mind guide can enable analyzer to pick up a review point of view on intricate, multifaceted ideas.

Vol 1 (3), September 2017, www.ijirase.com
Mind maps can likewise enable analyzer to diagram an article or story they're intending to compose. In the research paper implementing Collaborative mind mapping techniques. In The new innovative techniques performing testing, test case collaboration in this way to provide very effective and good result production and Problem Solving, Planning Activities, Structural Representations, Team Activities, Mind maps are flexible, Mind maps enable a free flow of ideas, Mind maps are visually domain driven, and Mind maps are clear structure of programming.

Key words: collaboration mind mapping, test case, traceability, Requirements.

\section{Introduction}

\subsection{Mind mapping}

Mind mapping is one of the approaches to study and keeping a dynamic personality as one takes part in preparing any new creative data. The proficiency of a shared personality mapping contemplating apparatus turns out to be increasingly effective relying upon its ability to keep 
the client associated with building up his basic considering and propelled to process new creative data and new thoughts. A mind mapping application enables the educator to make any sort of instructive portray or layout, which can be utilized as a beginning stage by analyzer. This technique for sharing essential substance empowers customized learning. Also, community oriented learning in This way, analyzers figure out how to think basically by making inquiries, requiring input, including remarks and rehearsing their own inventiveness as they add on new substance. Also, new imaginative deduction techniques exercises teammate in the learning procedure the quicker he will have the capacity to possess and decipher the conveyed data.

\subsection{Software Testing}

Software testing an investigation manages to provide business domain oriented in this information about the quality of the product and service under test. Software testing can also provide an objective, to provide independent view of the software to allow the business environment to appreciate and understand the risks of software implementation. The testing techniques insert but are not limited to use the process of executing a program or application with the aim of finding software bugs (errors or other defects). The software has deciding which software testing tools. And so on....

\subsection{Test case collaboration}

The collaboration test case is the best way to promote more frequent and open communication between testers and users is to introduce Behaviour-driven Data (BDD testing) the new methods, have use universal language to articulate the most pressing demands the user and end user. These actions the playing field for interacting testers and developers, allowing them to explicit their questions and concerns with comfort and flexibility testing and collaboration mind mapping test cases.

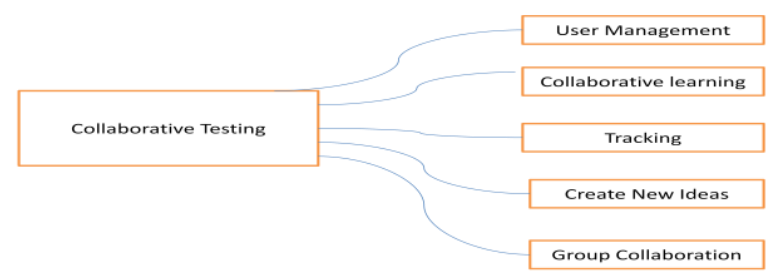

Fig-1

\subsection{Mind Map in Software Testing}

The software Testing is a very large area of creativity and thinking method every phase of software testing has its own methods and terminologies. It is the one of the individual where to apply 
collaborative mind map in software testing. It is always advisable to have good understanding and ground work of domestic branches of testing phase which you are planning to chalk out. The research is needed to collect all those thoughts into collaboration of one umbrella.

\section{2. RELATED WORK}

The paper Salzberg-Ludwig, Karin inquire about on mind maps in learning by rationally hindered kids Paper exhibited at the European Conference on Educational Research, University of Goteborg, 10-12 September 2008 [1]. The test chiefs abridged that the students of the exploratory gatherings worked more persuaded and all the more steadily and there were lesser conduct issues amid work. Compressing the topic with the assistance of catchphrases in a progressively requested structure made it feasible for the students of the trial gatherings to remember more learning for a more drawn out timeframe.

The creator Discussed in the Paper [9] Otaduy, I., and O. Diaz. "Client acknowledgment testing for Agile-created online applications: Empowering clients through wikis and mind maps." Journal of

Vol 1 (3), September 2017, www.ijirase.com
Systems and Software (2017). Agile methodologies put stringent demands on UAT, if only for the frequency at which it needs to be conducted. In-person meetings might need to be complemented with asynchronous ways for customers and developers to collaborate during UAT. We coin the term "self-paced UAT" to denote asynchronous sessions where customers perform UAT on their own using a scaffolding previously set by developers. Test scaffolding helps customers to effectively perform UAT (keeping the focus through testing hints) and efficiently (automatically setting customers in readyto-go scenarios through kickoffs). In addition, mind maps are proposed to give structure and context to UAT sessions. In this way, Record\&Replay is not launched in a vacuum but framed within a test map. First evaluations are promising. Subjects specially valued the chance of conducting UAT at their own pace. No travel, no agenda sync problems. They all prized the opportunity to add comments during test recording (the best rated feature in Likert scales) and to report feedback with a single click. Test parameterization was specially appreciated by the subjects who usually checked form-intensive websites.

In the Paper [11] Paul Farrand, Fearzana Hussain,Enid Hennessy Author 
portrayed The adequacy of the 'mind delineate' method Mind maps give a compelling examination strategy when connected to composed material. However before mind maps are by and large embraced as an investigation system, thought must be given towards methods for enhancing inspiration among clients.

In this paper [12] Author Karapanagiotidis, Theodoros, et al. "Tracking thoughts: Exploring the neural architecture of mental time travel during mindwandering." Neuroimage 147 (2017): 272281. Expanded actuations by positive valence in FR were in cerebrum locales pivotal to feeling acknowledgment and social collaboration. Expanded initiation of the premotor cortex may fill in as a compensatory instrument as FR subjects may need to apply more exertion on preparing the jolts, as has been discovered before in schizophrenia. Inability to deactivate PFC structures may suggest blunder in the default mode organize. Unusual PFC work in FR was additionally recommended by PPI,

\section{Collaboration Mind Mapping Methodology Frame Work}

\section{In this Research work implementing} new frame work contribution as Test

Vol 1 (3), September 2017, www.ijirase.com cases can easily user friendly be included in a collaboration mind map techniques since the maps are usually easy to maintain and changing requirements. The requirements will be represented without any difficulty. My suggestion would be to draw branches to every epic or user story and associate all the corresponding Functionalities as sub functionality. The tester can add test cases under each functionality and sub functionalities. The tester similar approach for creating an integration test for the application the testing team was working on. The map was continuously updated and maintained and every time, and every day before a deploy on the live environment we would run the integration test which consisted of multiple requirements. The research work add a corresponding image for PASS or FAIL to each flow and the entire team had access to the mind maps and test case map so they could see real time if our tests were passing and everything was all right. 


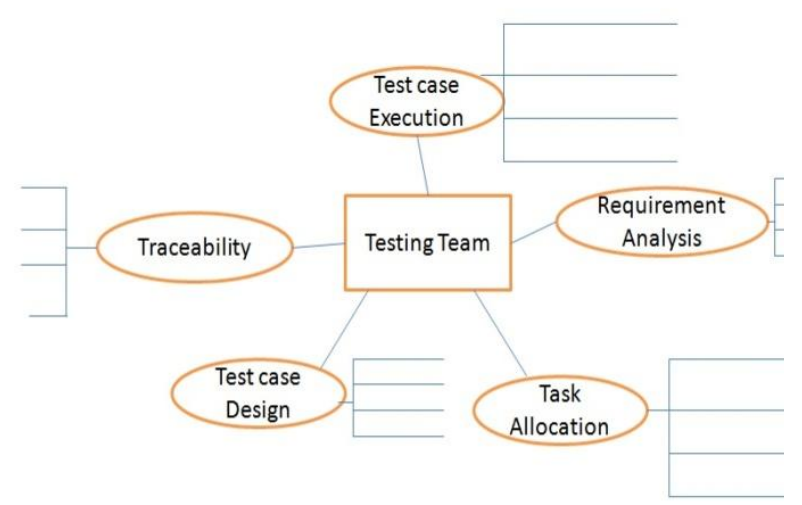

Fig-2

In the research work contribute the diagram collaborative mind mapping technique used and created maps substitute of writing test cases and got approval from clients, the client will give during crunch time. Also making the resources use this new innovative technique gives small interest in creating maps than usual test case design.

\subsection{Requirement Traceability Matrix} Mind Map

In this research paper implementing Traceability matrix test case collaborative mind mapping idea is more important for testing perspective. In this idea of mind mapping testing requirements test cases by preparing test traceability matrix. Through this techniques have to ensure that covered all the testing functionality of the testing Vol 1 (3), September 2017, www.ijirase.com application. The Traceability intermediate to requirements and other downstream components like tests, task, team, milestones and so on, achieved from collaborative mind mapping.

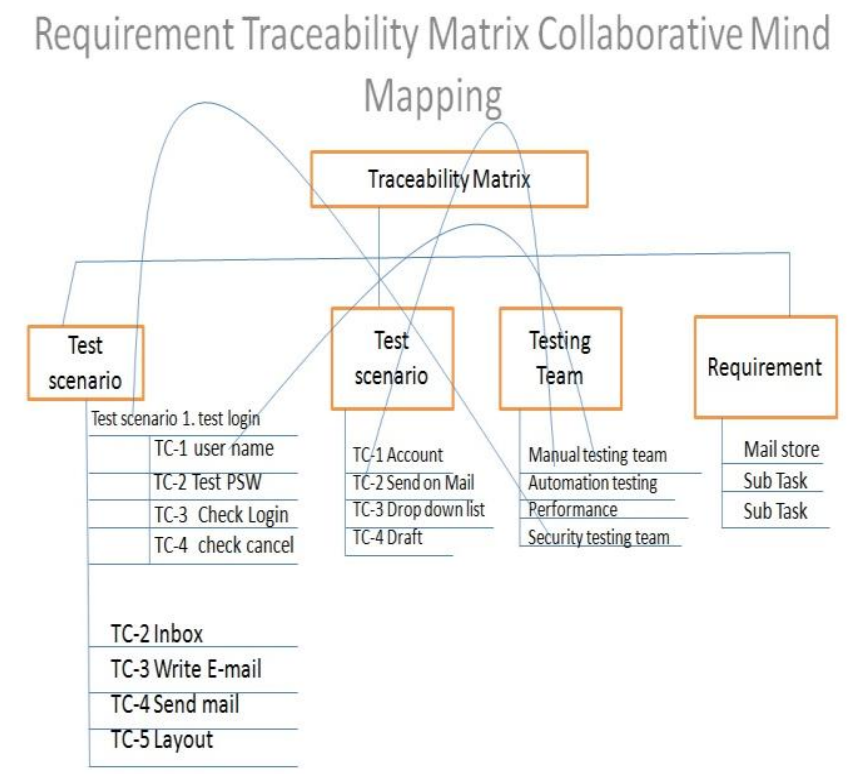

Fig -3

The above diagram of collaborative mind map the new innovative techniques to be used in any testing life cycle phase of the testing environment. And the research paper implementing many more scenarios tested at a same time in this research diagram.

The sample Test case creation from Use case / Requirements.

1. General test case report management techniques. 
2. Automation testing team script Management.

\section{References}

3. Testing Team management.

4. Daily meeting and weekly meeting, monthly meeting

5. Tester Reviews

6. Feedbacks forms ect...

1. Scholarly research on mind maps in learning by mentally retarded children Paper presented at the European Conference on Educational Research, University of Goteborg, 10-12 September 2008

\section{Concussion}

In this Research work success fully implementing collaboration mind mapping techniques using testing team easy to collaborative test cases, making a mind map is an very good and extraordinary way for able to sort through the testers' thoughts and concepts of the research contribution fast generate creative and even unique in less time, and this research method is a large way for Guggenheim and organize the ideas you brainstormed and identify their relationships and review your concepts fast and quick. There is a power of collaboration and test design is an active that advantage greatly from group interaction. Test Storming is where test design meets brainstorming to define creative and effective tests and test cases in this research paper to be successfully implementing create a good leader and good participants and a way to capture the input from multiple users and user interactions.

2. Salzberg-Ludwig, K.: Die Mind Mapping Methode und ihre Möglichkeiten zur Förderung von Gedächtnisleistungen bei lernbeeinträchtigten Schülerinnen und Schülern. In: SalzbergLudwig, K./ Grüning, E. (2007): Pädagogik für Kinder und Jugendliche in schwierigen Lernund Lebenssituationen. Stuttgart: Kohlhammer, S. 253-270

3. Mento, Anthony J., Patrick Martinelli, and Raymond M. Jones. "Mind mapping in executive education: applications and outcomes." Journal of Management Development 18.4 (1999): 390-416.

4. Davies, Martin. "Concept mapping, mind mapping and argument mapping: what are the differences and do they matter?." Higher education 62.3 (2011): 279-301.

5. Farrand, Paul, Fearzana Hussain, and Enid Hennessy. "The efficacy of the mind map 'study technique." Medical education 36.5 (2002): 426-431. 
6. Beel, Joeran, et al. "Utilizing mind-maps for information retrieval and user modelling." International Conference on User Modelling, Adaptation, and Personalization. Springer, Cham, 2014.

7. Al Naqbi, Shamma. "The use of mind mapping to develop writing skills in UAE schools." Education, Business and Society: Contemporary Middle Eastern Issues 4.2 (2011): 120-133.

8. Gordon, Anne CL, and David R. Olson. "The relation between acquisition of a theory of mind and the capacity to hold in mind." Journal of Experimental Child Psychology 68.1 (1998): 70-83.

9. Otaduy, I., and O. Diaz. "User acceptance testing for Agile-developed web-based applications: Empowering customers through wikis and mind maps." Journal of Systems and Software (2017).

10. Moran, Joseph M., et al. "Impaired theory of mind for moral judgment in highfunctioning autism." Proceedings of the National Academy of Sciences 108.7 (2011): 2688-2692.

11. Farrand, P., Hussain, F. and Hennessy, E. (2002), The efficacy of the 'mind map' study technique. Medical Education, 36: 426-431.doi:10.1046/j.13652923.2002.01205.x

12. Karapanagiotidis, Theodoros, et al. "Tracking thoughts: Exploring the neural architecture of mental time travel during mind-wandering." Neuroimage 147 (2017): 272-281. 